\title{
Aptamer Based Aflatoxin B1 Detection System Using Graphene Oxide Quencher
}

\author{
Min Young Joo, Seung Hoon Baek, Tae Jung Park \\ Chung-Ang University \\ Heukseok-ro 84, Dongjak-gu, Seoul, Korea \\ myjoo0406@gmail.com; cutey1221@naver.com; \\ tjpark@cau.ac.kr
}

\section{Extended Abstract}

Aflatoxin B1 is one of the most common mycotoxins, toxic substances produced by fungi, and produced by Aspergillus flavus. It is classified as one of International Agency for Research on Cancer (IARC) Group 1 carcinogens and only few nanograms of aflatoxin B1 may cause liver cancer and stunted growth by permeation through skin. There are conventional diagnosis methods such as high-performance liquid chromatography (HPLC) and immunoassays. These methods provide reliable quantification results but require well-trained professionals, expensive instruments and materials, and they are time consuming. In this research, nanomaterials, graphene oxide and aptamer, are used to overcome these weaknesses. Aptamer is a single-strand oligonucleotide, which binds to a target specifically and it is easy to synthesize. Aptamer specific for aflatoxin B1 is used as a probe and it is modified with a fluorescence dye, FAM on the 5' end. If the aptamer binds to an aflatoxin B1 molecule, the aptamer and aflatoxin B1 complex becomes easy to interact with graphene oxide, a well-known fluorescence quencher. It results in the decrease of the fluorescence intensity, and the fluorescence intensity increases with the lack of aflatoxin B1, on the other hand.

Graphene oxide was synthesized by the general method, Hummer's method. The FAM modified aptamer and aflatoxin B1 from A. flavus were purchased. The detecting process was conducted through 3 steps. The aptamer was mixed with aflatoxin B1 and incubated for minutes in the first step. Then, graphene oxide was added to the mixture and incubated for few minutes again to collect the unbound aptamer molecules. At the final step, the fluorescence intensity was measured. The concentration of aptamer was determined by few tests, and then the graphene oxide concentration was optimized to show the best quenching efficiency. In addition, the incubation time and temperature for quenching and binding was optimized, respectively. The detection system was evaluated with standard solutions of aflatoxin B1. The results showed that the fluorescence intensity linearly decreased and was dependent on the concentration of aflatoxin B1. It indicates a possibility of detecting the target quantitatively with this method. The limit of detection was lower than the permitted level of aflatoxin B1 within food, which was determined by World Health Organization (WHO). Moreover, the whole detection procedure took less than an hour.

The aflatoxin B1 detection system using aptamer and graphene oxide is rapid, simple and cost effective. Hence, it is concerned to be a competitive detection system on the field compared to the conventional detection methods. Besides, the system can also be applied to other mycotoxins if the probe sequences of aptamers are replaced, specific to the targets.

\section{References}

[1] Z. Lu, "Aptamer based fluorescence recovery assay for aflatoxin B1 using a quencher system composed of quantum dots and graphene oxide," Microchim. Acta. vol. 182, pp. 571-578, 2015.

[2] H. Y. Jeong, "Robust fluorescence sensing platform for detection of CD44 cells based on graphene oxide/gold nanoparticles," Colloid. Surface B, vol. 135, pp. 309-315, 2015. 